\title{
Fragility analysis of bridges under ground motion with spatial variation
}

\author{
Sang-Hoon Kim*, Maria Q. Feng \\ Civil and Environmental Engineering, University of California, Irvine, CA USA
}

\begin{abstract}
Seismic ground motion can vary significantly over distances comparable to the length of a majority of highway bridges on multiple supports. This paper presents results of fragility analysis of highway bridges under ground motion with spatial variation. Ground motion time histories are artificially generated with different amplitudes, phases, as well as frequency contents at different support locations. Monte Carlo simulation is performed to study dynamic responses of an example multi-span bridge under these ground motions. The effect of spatial variation on the seismic response is systematically examined and the resulting fragility curves are compared with those under identical support ground motion. This study shows that ductility demands for the bridge columns can be underestimated if the bridge is analyzed using identical support ground motions rather than differential support ground motions. Fragility curves are developed as functions of different measures of ground motion intensity including peak ground acceleration, peak ground velocity, spectral acceleration, spectral velocity and spectral intensity. This study represents a first attempt to develop fragility curves under spatially varying ground motion and provides information useful for improvement of the current seismic design codes so as to account for the effects of spatial variation in the seismic design of long-span bridges. (C) 2002 Elsevier Science Ltd. All rights reserved.
\end{abstract}

Keywords: Fragility curve; Concrete bridge; Nonlinear dynamic analysis; Earthquake; Ductility

\section{Introduction}

Fragility curves find their modern origin in the seismic probabilistic risk assessment of nuclear power plants performed in the early seventies. Simply stated, they characterize seismic capacity of mechanical and structural systems in the form of a probability distribution function, usually as a function of the ground motion intensity. The measures such as peak ground acceleration (PGA), peak ground velocity (PGV), spectral acceleration (SA), spectral velocity (SV) and spectral intensity (SI) are often deployed to represent the intensity, and the probability distribution function is used to reflect uncertain factors involved in these intensity measures as well as in the structural capacity.

While performing a seismic risk analysis of highway system, it is imperative to identify seismic vulnerability associated with various damage states of bridges, since the bridges are among the most seismically vulnerable structures in the system. The fragility curve of a bridge, representing its seismic vulnerability information, is traditionally defined as the probability that the structure under consideration will suffer from physical damage

\footnotetext{
* Corresponding author.
} 
in a specific state upon subjected to an earthquake ground motion of a given intensity level. In principle, the development of bridge fragility curves requires synergetic use the following approaches: (1) professional judgement, (2) quasi-static and design code consistent analysis, (3) utilization of damage data associated with past earthquakes and (4) numerical simulation of seismic response based on structural dynamics.

More recently, a number of studies on fragility curves for highway bridges were made along these lines [1-4]. Most of them, whether empirical or analytical, were based on the assumption that the structure under consideration is subjected to an identical ground motion. However, a majority of multi-span bridges are likely to suffer ground motions at their supports that can differ considerably in amplitude, phases as well as frequency content, since seismic ground motion can vary significantly over distances comparably to the length of the bridge. The collapse of the 463-m long bridge in the SR14/I5 interchange during the 1994 Northridge earthquake is an example suggesting that the effects of the spatial variation of the ground motion might have caused the failure considering the length of the bridge and the different soil conditions at the locations of the various supports [5].

A preliminary investigation was performed earlier by Shinozuka et al. [6] on seven typical California bridge models, through which it was found that for several of the bridges the differential support ground motion produces significantly higher structural response than the identical support ground motion. As a result, the assumption of identical support ground motion is unconservative in that peak ductility demand for columns would be underestimated, if the bridges were to be analyzed using identical support ground motion, rather than differential support ground motion. Therefore, it is of paramount importance to account for the effect of spatial variation of earthquake ground motion in developing fragility curves for highway bridges, particularly for multi-span long bridges.

It should be mentioned that fragility curves for bridges on multiple supports accounting for the effect of spatial variation of seismic ground motion (including different local soil conditions) were developed first by Saxena [7], Deodatis, Saxena and Shinozuka [8], and Saxena, Deodatis, Shinozuka and Feng [9] for ground motion acting along the axis of the bridge. This paper extends the aforementioned work to account for ground motion acting perpendicular to the axis of the bridge.

\section{Generation of seismic ground motion with spatial variation}

The spatial variation of seismic ground motion can be attributed to the following three mechanisms [10]: (1) the difference in arrival times of the seismic waves at different locations, commonly known as the "wave passage effect", (2) the change in shape of the propagating waveform due to multiple scatterings of the seismic waves in the highly inhomogeneous soil medium, referred to as the "incoherence effect", and (3) the change in amplitude and frequency content of ground motion at different locations on the ground surface due to different local soil conditions known as the "local site effect".

In this paper, an iterative algorithm proposed by Saxena [7] and Deodatis [11] has been used to generate differential acceleration time histories at several prescribed locations on the ground surface. The methodology is described as follows, by considering that the acceleration time histories at a specified number of locations on the ground surface constitute a multi-variate, non-stationary, stochastic process (non-stationary stochastic vector process).

\subsection{Simulation of n-variate non-stationary stochastic processes}

This section outlines the algorithm which simulates non-stationary ground motion time histories based on a prescribed spectral density matrix. The vector process is assumed to be a non-stationary vector process with evolutionary power. To be specific, consider a $n$-variate, non-stationary stochastic vector process with components $f_{j}^{0}(t) ; j=1,2, \ldots, n$, having mean value equal to zero i.e. $\varepsilon\left[f_{i}^{0}(t)\right]=0 ; j=1,2, \ldots, n$ and cross-spectral 
density matrix given by

$$
S^{0}(\omega, t)=\left[\begin{array}{cccc}
S_{11}^{0}(\omega, t) & S_{12}^{0}(\omega, t) & \cdots & S_{1 n}^{0}(\omega, t) \\
S_{21}^{0}(\omega, t) & & & \vdots \\
\vdots & & \ddots & \vdots \\
S_{n 1}^{0}(\omega, t) & \ldots & \ldots & S_{n n}^{0}(\omega, t)
\end{array}\right] .
$$

Due to the assumed non-stationarity of the vector process, the cross-spectral density matrix in Eq. (1) will be a function of both frequency $\omega$ and time $t$. For the purpose of this study, a special case of Eq. (1) is assumed to hold:

$$
\begin{aligned}
& S_{j j}^{0}(\omega, t)=\left|A_{j}(t)\right|^{2} S_{j}(\omega), \quad j=1,2, \ldots, n, \\
& S_{j k}^{0}(\omega, t)=A_{j}(t) A_{k}(t) \sqrt{S_{j}(\omega) S_{k}(\omega)} \Gamma_{j k}(\omega), \quad j, k=1,2, \ldots, n, \quad j \neq k,
\end{aligned}
$$

where $A_{j}(t) ; j=1,2, \ldots, n$ are the modulating functions of the vector process $f_{j}^{0}(t) ; j=1,2, \ldots, n$ and $S_{j}(\omega) ; j=1,2, \ldots, n$ are the corresponding (stationary) power spectral density functions. The functions $\Gamma_{j k}(\omega)$; $j, k=1,2, \ldots, n ; j \neq k$ are the complex coherence functions describing the correlation structure between the components of the stationary vector process. They are given by

$$
\Gamma_{j k}(\omega)=\gamma_{j k}(\omega) \exp \left[-\mathrm{i} \frac{\omega \xi_{j k}}{v}\right], \quad j, k=1,2, \ldots, n, j \neq k,
$$

where $\gamma_{j k}(\omega) ; j, k=1,2, \ldots, n ; j \neq k$ are the (stationary) coherence functions between $f_{j}^{0}(t)$ and $f_{k}^{0}(t)$. $\exp [-$ $\left.\mathrm{i}\left(\omega \xi_{j k} / v\right)\right]$ is the wave propagation term where $\xi_{j k}(\omega)$ is the distance between points $j$ and $k$, and $v$ is the velocity of wave propagation.

Given the simple structure of Eqs. (2)-(3), where the modulating function is deterministic, the components of the non-stationary process $f_{j}^{0}(t) ; j=1,2, \ldots, n$ can be expressed as a product of a zero mean stationary process $g_{j}^{0}(t) ; j=1,2, \ldots, n$ and the respective modulating function, i.e.

$$
\begin{aligned}
& f_{j}^{0}(t)=A_{j}(t) g_{j}^{0}(t), \quad j=1,2, \ldots, n, \\
& \varepsilon\left[g_{j}^{0}(t)\right]=0, \quad j=1,2, \ldots, n .
\end{aligned}
$$

From Eqs. (2), (3) and (5), the cross-spectral density matrix for the stationary process $g_{j}^{0}(t) ; j=1,2, \ldots, n$ is given by

$$
\mathbf{S}^{0}(\omega)=\left[\sqrt{S_{j}(\omega) S_{k}(\omega)} \Gamma_{j k}(\omega)\right], \quad j, k=1,2, \ldots, n,
$$

where we have adopted the convention $\Gamma_{j j}=1$ and used the notation in Eqs. (2) and (3).

\subsection{Simulation procedure}

In order to simulate samples of the $n$-variate non-stationary stochastic process $f_{j}^{0}(t) ; j=1,2, \ldots, n$, its (stationary) cross-spectral density matrix $\mathbf{S}^{0}(\omega)$ given in Eq. (7) is factorized into the following product:

$$
\begin{aligned}
& \mathbf{S}^{0}(\omega)=\mathbf{H}(\omega) \mathbf{H}^{\mathrm{T} *}(\omega), \\
& \mathbf{H}(\omega, t)=\left[\begin{array}{cccc}
H_{11}(\omega) & & & \\
H_{21}(\omega) & H_{22}(\omega) & & \\
\vdots & \vdots & \ddots & \\
H_{n 1}(\omega) & \cdots & \cdots & H_{n n}(\omega)
\end{array}\right]
\end{aligned}
$$


using Cholesky's decomposition method. The diagonal elements of $\mathbf{H}(\omega)$ are real and non-negative functions of $\omega$, while the off-diagonal elements are generally complex functions of $\omega$. The elements of $\mathbf{H}(\omega)$ can be written in polar form as

$$
H_{j k}(\omega)=\left|H_{j k}(\omega)\right| \mathrm{e}^{\mathrm{i} \theta_{j k}(\omega)}, \quad j>k,
$$

where

$$
\theta_{j k}(\omega)=\tan ^{-1}\left(\frac{\operatorname{Im}\left[H_{j k}(\omega)\right]}{\operatorname{Re}\left[H_{j k}(\omega)\right]}\right) .
$$

Once the matrix $\mathbf{S}^{0}(\omega)$ is decomposed according to Eqs. (8)-(10), the stationary stochastic vector process $g_{j}^{0}(t) ; j=1,2, \ldots, n$ can be simulated by the following series as $N \rightarrow \infty$

$$
g_{j}(t)=2 \sum_{m=1}^{n} \sum_{l=1}^{N}\left|H_{j m}\left(\omega_{l}\right)\right| \sqrt{\Delta \omega} \quad \cos \left[\omega_{l} t-\theta_{j m}\left(\omega_{l}\right)+\Phi_{m l}\right], \quad j=1,2, \ldots, n,
$$

where

$$
\begin{aligned}
& \omega_{l}=l \Delta \omega, \quad l=1,2, \ldots, N, \\
& \Delta \omega=\frac{\omega_{\mathrm{u}}}{N}, \\
& \theta_{j m}\left(\omega_{l}\right)=\tan ^{-1}\left(\frac{\operatorname{Im}\left[H_{j m}\left(\omega_{l}\right)\right]}{\operatorname{Re}\left[H_{j m}\left(\omega_{l}\right)\right]}\right) .
\end{aligned}
$$

The quantities $\left\{\Phi_{m l}\right\} ; m=1,2, \ldots, n ; l=1,2, \ldots, N$ appearing in Eq. (12) are $n$ sequences of independent random phase angles distributed uniformly over the interval $[0,2 \pi]$. In Eq. (14), $\omega_{\mathrm{u}}$ represents an upper cut-off frequency beyond which the elements of the cross-spectral density matrix in Eq. (1) may be assumed to be zero for any time instant $t$. As such, $\omega_{\mathrm{u}}$ is a fixed value and hence $\Delta \omega \rightarrow 0$ as $N \rightarrow \infty$, so that $N \Delta \omega=\omega_{\mathrm{u}}$.

In order to generate the $i$ th sample $g_{j}^{(i)}(t) ; j=1,2, \ldots, n$ of the stationary stochastic vector process $g_{j}(t) ; j=$ $1,2, \ldots, n$, one replaces the $n$ sequences of random phase angles $\left\{\Phi_{m l}\right\} ; m=1,2, \ldots, n ; l=1,2, \ldots, N$ in Eq. (12) with their respective $i$ th realizations $\left\{\phi_{m l}\right\} ; m=1,2, \ldots, n ; l=1,2, \ldots, N$ :

$$
g_{j}^{(i)}(t)=2 \sum_{m=1}^{n} \sum_{l=1}^{N}\left|H_{j m}\left(\omega_{l}\right)\right| \sqrt{\Delta \omega} \times \cos \left[\omega_{l} t-\theta_{j m}\left(\omega_{l}\right)+\phi_{m l}^{(i)}\right], \quad j=1,2, \ldots, n .
$$

The corresponding $i$ th realization of the non-stationary vector process $f_{j}^{(i)}(t) ; j=1,2, \ldots, n$ is calculated by multiplying the $i$ th realization of the stationary process $g_{j}^{(i)}(t) ; j=1,2, \ldots, n$ by the modulating functions $A_{j}(t) ; j=1,2, \ldots, n$ :

$$
f_{j}^{(i)}(t)=A_{j}(t) g_{j}^{(i)}, \quad j=1,2, \ldots, n .
$$

\subsection{Simulation of ground motion compatible with prescribed response spectra}

An iterative algorithm shown in Table 1 is used to generate acceleration time histories at $n$ points on the ground surface that are compatible with prescribed response spectra. A different target acceleration response spectrum $\operatorname{RSA}_{i}(\omega) ; i=1,2, \ldots, n$ can be assigned to each of these points, since those points can generally be on different local soil conditions. Complex coherence functions $\Gamma_{j k}(\omega) ; j, k=1,2, \ldots, n ; j \neq k$ are prescribed between pairs of points and modulating functions $A_{j}(t) ; j=1,2, \ldots, n$ are assigned at each point. The power spectral density functions $S_{j}(\omega) ; j=1,2, \ldots, n$ in the first iteration are initialized to a constant (non-zero) value over the entire frequency range. After setting up the cross-spectral density matrix given in Eq. (7) according 
Table 1

Iterative scheme to generate acceleration time histories compatible response spectrum at $n$ points on the ground surface

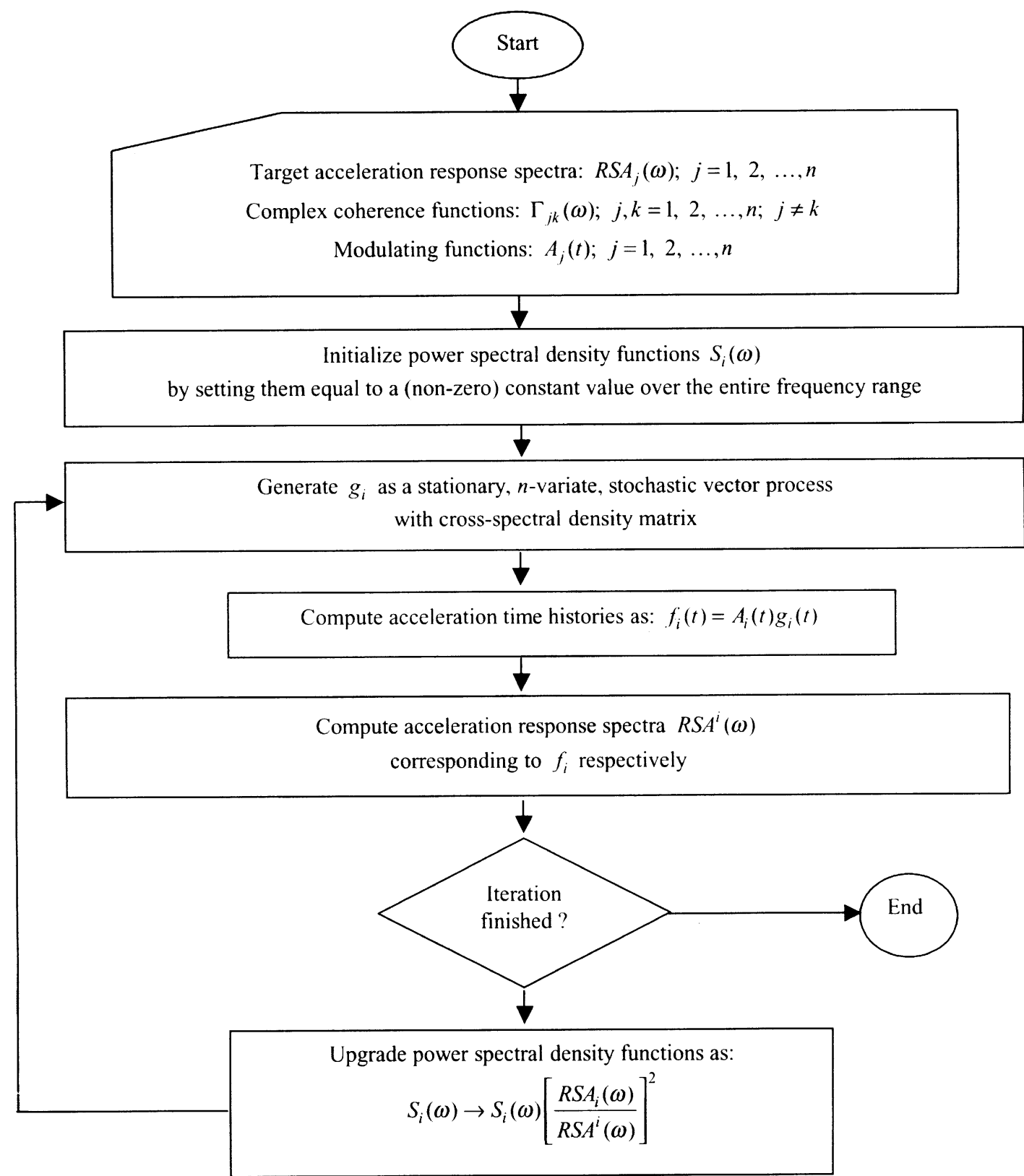



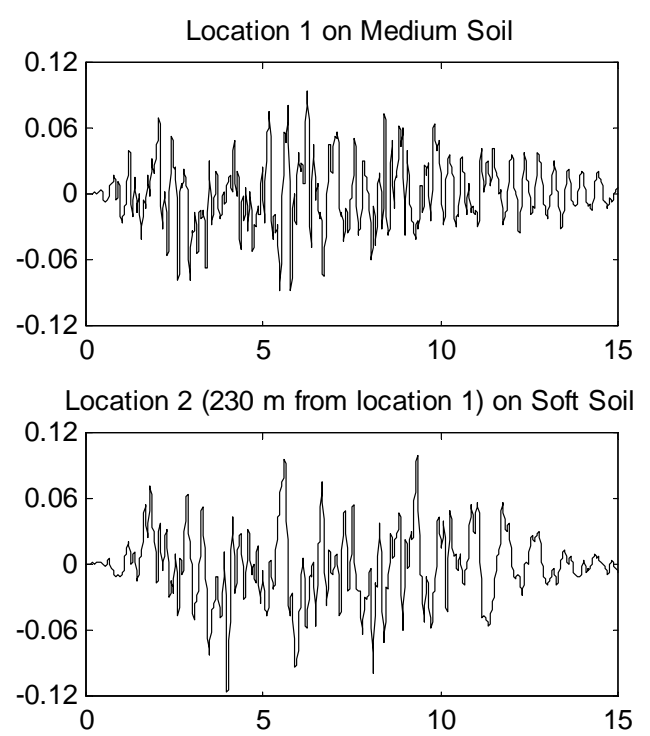

Location 3 (460 m from location 1) on Medium Soil

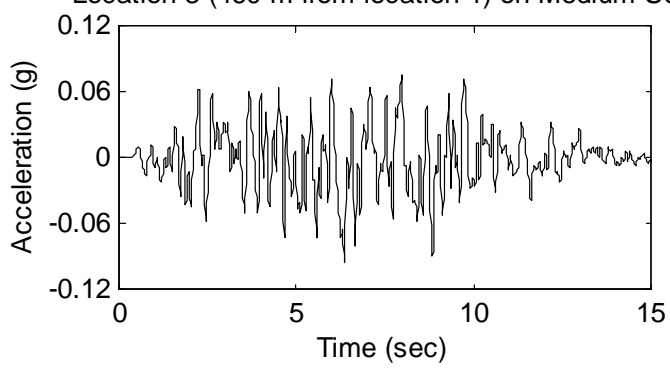

Fig. 1. Acceleration time histories.

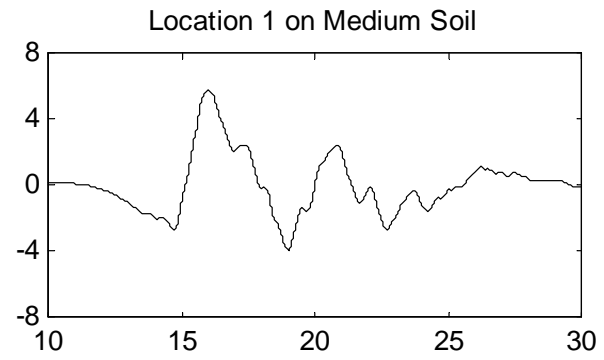

Location 2 (230 m from location 1) on Soft Soil

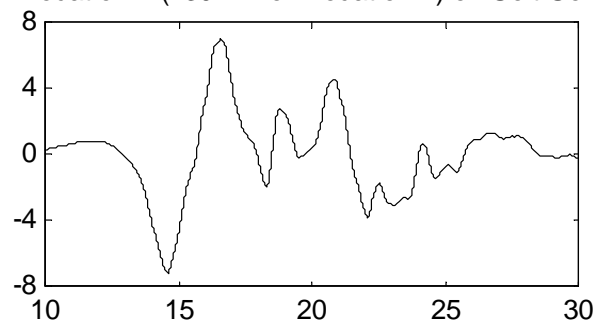

Location 3 (460 m from location 1) on Medium Soil

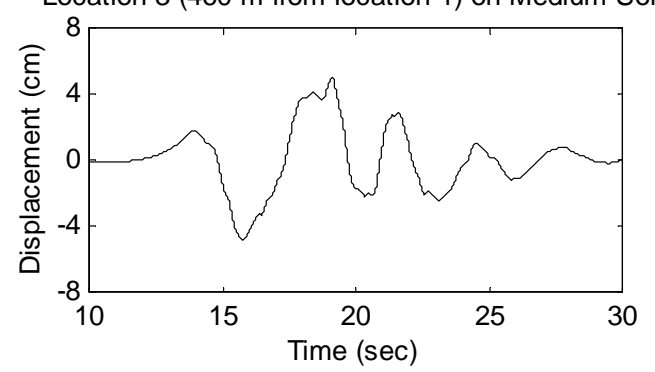

Fig. 2. Displacement time histories.

to a prescribed coherence function and a velocity of wave propagation, the stationary ground motion time histories are generated using the simulation formula given in Eq. (12). The non-stationarity is then introduced by multiplying each of the stationary time histories with modulating functions $A_{j}(t) ; j=1,2, \ldots, n$. In the next step, the response spectra of the simulated non-stationary time histories are calculated and matched with the prescribed response spectra. In case, the response spectra do not match at a chosen level of accuracy, the diagonal terms of the cross-spectral density matrix of the underlying stationary are upgraded as shown in Table 1.

Asynchronous acceleration and displacement time histories at three different locations were shown in Figs. 1 and 2, respectively.

\section{Effect of spatial variation on bridge response}

This section briefly reviews the work done by Shinozuka et al. [6]. The effect of spatial variation of seismic ground motion on bridge response was investigated through Monte Carlo simulation of response time histories of sample bridges in both longitudinal and transverse directions under differential ground motions. Seven 
Table 2

Seven sample bridges

\begin{tabular}{|c|c|c|c|c|}
\hline Bridge & Total length (m) & Number of spans & Number of expansion joints & Natural period (s) \\
\hline TEXT & 34 & 3 & 0 & $0.77^{\mathrm{a}} 0.18^{\mathrm{b}}$ \\
\hline FHWA2 & 122 & 3 & 0 & $0.92^{\mathrm{a}} 0.81^{\mathrm{b}}$ \\
\hline TYOH & 242 & 5 & 0 & $1.42^{\mathrm{a}} 1.71^{\mathrm{b}}$ \\
\hline TY1H & 242 & 5 & 1 & $1.52^{\mathrm{a}} 1.96^{\mathrm{b}}$ \\
\hline TY2H & 242 & 5 & 2 & $1.69^{\mathrm{a}} 1.98^{\mathrm{b}}$ \\
\hline GC2D & 244 & 5 & 2 & $1.19^{\mathrm{a}} 0.74^{\mathrm{b}}$ \\
\hline Santa Clara & 500 & 12 & 1 & $0.59^{\mathrm{a}} 0.84^{\mathrm{b}}$ \\
\hline
\end{tabular}

\footnotetext{
${ }^{\text {a}}$ For longitudinal direction.
}

${ }^{\mathrm{b}}$ For transverse direction.

sample bridges shown in Table 2, representing typical California highway bridges, were selected with total lengths ranging from $34-\mathrm{m}$ up to $500-\mathrm{m}$, and with number of spans ranging from 3 up to 12 . Non-linear dynamic time history analyses were performed using the computer code SAP2000 Non-linear version [12]. Each of the selected bridges was subjected to a number of earthquakes, each containing 20 different sets of ground motion time histories. For each scenario earthquake, the bridge under consideration was analyzed using identical and differential support ground motions. The ratio $\rho$ defined as follows was then computed for each bridge in order to quantify the effect of the spatial variation of ground motion on the response of the bridge

$$
\rho=\frac{\max \text { of response quantity computed using differential support ground motion }}{\text { max of same quantity computed using identical support ground motion }} .
$$

The parameter used to describe the non-linear structural response in this study is the ductility demand. The ductility demand is defined as $\theta / \theta_{\mathrm{y}}$, where $\theta$ is the rotation of a bridge column in its plastic hinge and $\theta_{\mathrm{y}}$ is the corresponding rotation at the yield point. The non-linear model of a column is depicted in Fig. 3.

From the results of the Monte Carlo simulation using the ground displacement time histories generated in this study as inputs, the ratio of the mean value of the ductility demands computed under ground motions without and with spatial variation is plotted in Fig. 4, respectively, for the longitudinal and transverse directions as functions of the bridge length. It is demonstrated that the ductility demand increases substantially when the bridge is subjected to differential ground motion, compared to that under identical ground motion. It is also indicated that the transverse structural response of TEXT bridge is very sensitive to the soil condition. Especially, this increase in ductility ratio for both longitudinal and transverse direction is of the order 1.432.5 for the five medium span bridges, and as high as 4.0 for the longest Santa Clara bridge. Therefore, for bridges more than $300 \mathrm{~m}$ in total length and/or bridges with supports on different local soil conditions, it is recommended to perform time history dynamic analyses for the design purpose, using differential ground motion time histories.

\section{Development of fragility curves}

A logical extension of the work performed by Shinozuka et al. [6] and briefly reviewed in Section 3 is to develop fragility curves for these bridges accounting for the effect of the spatial variation of the ground 


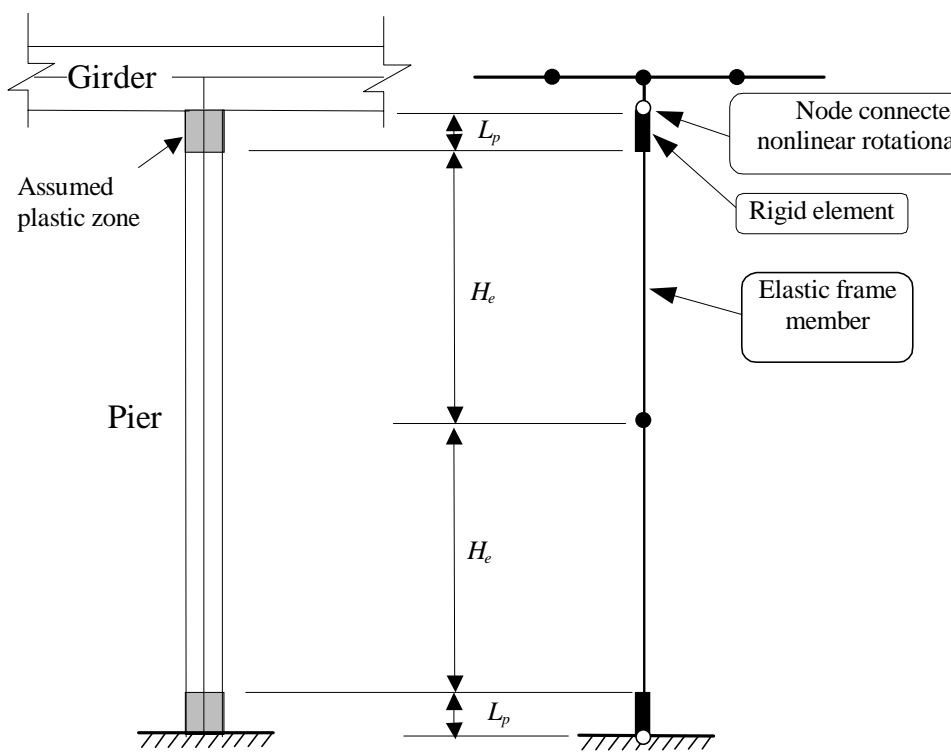

(a) (b)

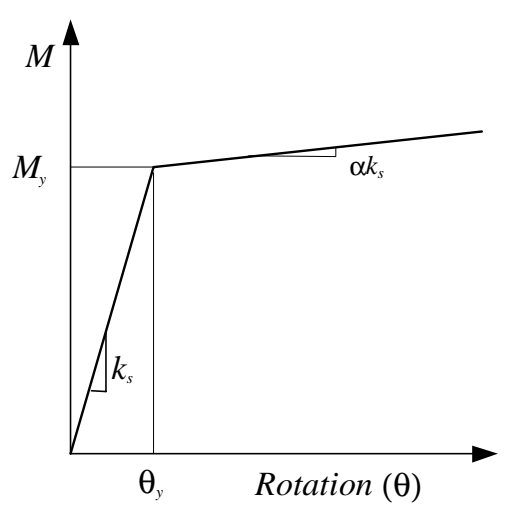

(c)

Fig. 3. Non-linear model for bridge column. (a) Model of column, (b) finite element model of column and (c) moment-curvature relationship of non-linear rotational spring.
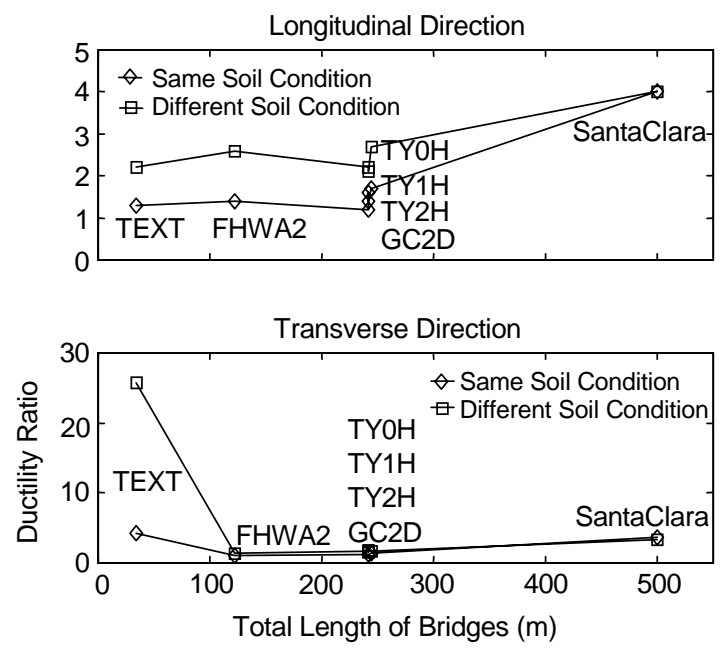

Fig. 4. Ratio of maximum ductility demands of sample bridges.

motion input. As a first attempt to achieve this, the present study develops the fragility curves for the Santa Clara bridge subjected to the ground motion with spatial variation.

The approach for generating empirical fragility curves was explained through an example using Caltrans bridges [3]. A family of four fragility curves can, for example, be developed independently for the damage states, respectively, identified as "at least minor", "at least moderate", "at least major" and "collapse", making 
use of the entire sample (of size equal to 1998) of Caltrans' expressway bridges in Los Angeles County, California subjected to the Northridge earthquake and inspected for damage after the earthquake. It is assumed that the curves can be expressed in the form of two-parameter of log-normal distribution functions, and the estimation of the two parameters (median and log-standard deviation) is performed with the aid of the maximum likelihood method. For this purpose, the PGA is used to represent the intensity of the seismic ground motion, although intensity measures other than PGA such as PGV, SA, SV and SI can also be used for development of fragility curves.

The likelihood function for the present purpose is expressed as

$$
L=\prod_{i=1}^{N}\left[F\left(a_{i}\right)\right]^{x_{i}}\left[1-F\left(a_{i}\right)\right]^{1-x_{i}},
$$

where $F($.$) represents the fragility curve for a specific state of damage, a_{i}$ is the PGA value to which bridge $i$ is subjected, $x_{i}$ represents realizations of the Bernoulli random variable $X_{i}$ and $x_{i}=1$ or 0 depending on whether or not the bridge sustains the state of damage under PGA $=a_{i}$, and $N$ is the total number of bridges inspected after the earthquake. Under the current log-normal assumption, $F(a)$ takes the following analytical form

$$
F(a)=\Phi\left[\frac{\ln (a / c)}{\varsigma}\right]
$$

in which " $a$ " represents PGA and $\Phi[$.$] is the standardized normal distribution function.$

The two parameters $c$ and $\zeta$ in Eq. (19) are computed as $c_{0}$ and $\zeta_{0}$ satisfying the following equations to maximize in $\ln L$ and hence $L$;

$$
\frac{\mathrm{d} \ln L}{\mathrm{~d} c}=\frac{\mathrm{d} \ln L}{\mathrm{~d} \varsigma}=0
$$

This computation is performed by implementing a straightforward optimization algorithm.

Integrating the damage state information with that of the PGA, and making use of the maximum likelihood method involving Eqs. (18)-(20), four fragility curves can be constructed.

As the common sense dictates, the value of the fragility curve at a specified ground motion intensity such as PGA is always larger for a lesser state of damage than that for a severer state. In order to force the fragility curves not to intersect, a common log-standard deviation is estimated along with the medians of the log-normal distributions with the aid of the maximum likelihood method. The following likelihood formulation described by Shinozuka et al. [13] is introduced for the purpose of this method.

Although this method can be used for any number of damage states, it is assumed here for the ease of demonstration of analytical procedure that there are four states of damage including the state of no damage. A family of three (3) fragility curves exists in this case where events $E_{1}, E_{2}, E_{3}$ and $E_{4}$, respectively, indicate the state of no, at least minor, at least moderate and major damage. $P_{i k}=P\left(a_{i}, E_{k}\right)$ in turn indicates the probability that a bridge $i$ selected randomly from the sample will be in the damage state $E_{k}$ when subjected to ground motion intensity expressed by $\mathrm{PGA}=a_{i}$. All fragility curves are represented by two-parameter log-normal distribution functions

$$
F_{j}\left(a_{j} ; c_{j}, \varsigma_{j}\right)=\Phi\left[\frac{\ln \left(a_{i} / c_{j}\right)}{\varsigma_{j}}\right],
$$

where $c_{j}$ and $\zeta_{j}$ are the median and $\log$-standard deviation of the fragility curves for the damage state of "at least minor", "at least moderate" and "major" identified by $j=1,2$ and 3 . From this definition of fragility curves, and under the assumption that the log-standard deviation is equal to $\zeta$ common to all the fragility 


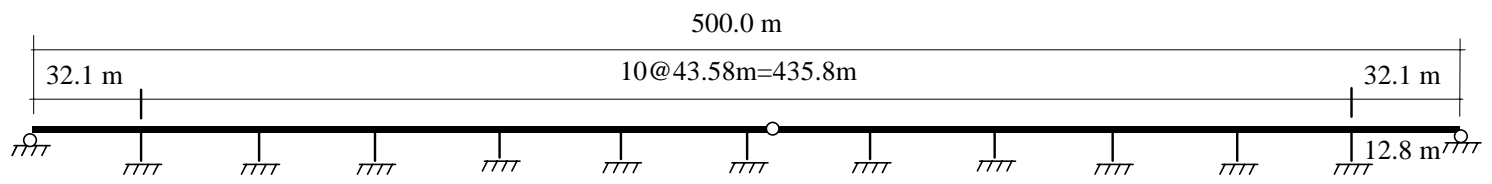

Fig. 5. Elevation of Santa Clara bridge.

curves, one obtains;

$$
\begin{aligned}
& P_{i 1}=P\left(a_{i}, E_{1}\right)=1-F_{1}\left(a_{i} ; c_{1}, \varsigma\right), \\
& P_{i 2}=P\left(a_{i}, E_{2}\right)=F_{1}\left(a_{i} ; c_{1}, \varsigma\right)-F_{2}\left(a_{i} ; c_{2}, \varsigma\right), \\
& P_{i 3}=P\left(a_{i}, E_{3}\right)=F_{2}\left(a_{i} ; c_{2}, \varsigma\right)-F_{2}\left(a_{i} ; c_{3}, \varsigma\right), \\
& P_{i 4}=P\left(a_{i}, E_{4}\right)=F_{3}\left(a_{i} ; c_{3}, \varsigma\right) .
\end{aligned}
$$

The likelihood function can then be introduced as

$$
L\left(c_{1}, c_{2}, c_{3}, \varsigma\right)=\prod_{i=1}^{n} \prod_{k=1}^{4} P_{k}\left(a_{i} ; E_{k}\right)^{x_{i k}},
$$

where

$$
x_{i k}=1
$$

if the damage state $E_{k}$ occurs the $i$ th bridge subjected to $a=a_{i}$, and

$$
x_{i k}=0
$$

otherwise. Then the maximum likelihood estimates $c_{0 j}$ for $c_{j}$ and $\zeta_{0}$ for $\zeta$ are obtained by solving the following equations:

$$
\begin{gathered}
\frac{\partial \ln L\left(c_{1}, c_{2}, c_{3}, \varsigma\right)}{\partial c_{j}}=\frac{\partial \ln L\left(c_{1}, c_{2}, c_{3}, \varsigma\right)}{\partial \varsigma}=0 \\
(j=1,2,3)
\end{gathered}
$$

by again implementing a straightforward optimization algorithm.

\subsection{Description of example bridge}

To demonstrate the development of analytical fragility curves, the twelve-span precast box girder Santa Clara bridge is used, which is the longest among the seven sample bridges. As shown in Fig. 5, the total length of the bridge is $500 \mathrm{~m}$ (two side spans $=32.1 \mathrm{~m}$ and other spans $=43.58 \mathrm{~m}$ ) and the height is $12.8 \mathrm{~m}$.

Along the lines of a Monte Carlo simulation, a total of 300 earthquakes without and with spatial variation were generated for the 11 supports of the bridge by considering five different PGA $(0.1,0.2,0.3,0.4$ and $0.5 \mathrm{~g}$ ), and varying the seed for 60 different random numbers for each PGA value. For each set of differential support ground motion time histories, the corresponding set of identical support ground motion time histories is obtained by considering that the ground motion time history at the first support of the bridge is applied at all the other supports. These earthquakes are briefly described in Table 3. The computer code SAP 2000/Non-linear was utilized in order to simulate the state of damage of the structure under ground displacement time histories without and with spatial variation. 
Table 3

Generated earthquakes

\begin{tabular}{llr}
\hline & Longitudinal direction & Transverse direction \\
\hline Range of simulated PGA $(\mathrm{g})$ & $0.023-0.725$ & $0.023-0.685$ \\
Soil & & $\begin{array}{l}\text { Three mid supports on soft soil and } \\
\text { eight supports on medium soil condition }\end{array}$ \\
Number of earthquakes simulated & 300 & 300 \\
\hline
\end{tabular}

Table 4

Damage states recommended by Dutta and Mander [14]

\begin{tabular}{lllll}
\hline Damage state & Description & $\begin{array}{l}\text { Drift limits } \\
\text { (non-seismically } \\
\text { designed) }\end{array}$ & $\begin{array}{l}\text { Drift limits } \\
\text { seismically } \\
\text { designed) }\end{array}$ & $\begin{array}{l}\text { Ductility demand } \\
\text { transformed from the } \\
\left.\text { drift limits, } \theta / \theta_{\mathrm{y}}\right)\end{array}$ \\
\hline No damage & First yield $\left(\theta_{\mathrm{y}}\right)$ & 0.005 & 0.008 & 1.00 \\
Slight damage & Cracking, spalling & 0.007 & 0.01 & 2.01 \\
Moderate damage & Loss of anchorage & 0.015 & 0.025 & 6.03 \\
Extensive damage & $\begin{array}{l}\text { Incipient column } \\
\text { collapse }\end{array}$ & 0.025 & 0.05 & 11.07 \\
Complete collapse & Column collapse & 0.05 & 0.075 & 23.65 \\
\hline
\end{tabular}

\subsection{Measures of damage state}

Damage of bridges due to earthquakes has been observed to occur in various degrees varying from light damage to collapse. In most cases, structural damage due to earthquakes can be attributed to excessive rotation of the plastic hinges formed at the columns of the bridge. It has been observed that a reinforced concrete bridge column subjected to lateral earthquake loading usually experiences progressive reduction in strength and stiffness due to inelastic actions. Such actions usually begin with yielding followed by bond failure in anchorage or lap slices or even shear failure in extreme situations. The severity of such effects is largely dependent on detailing, particularly on the transverse confining reinforcement both in potential plastic hinge zones and foundation/cap beam joints. The ductility that is derived from the rotation in the plastic hinge formed at the end of a column is considered to be a good measure of the damage. Therefore, in this study, the limit state is defined in terms of ductility demand of the columns of the bridge.

For the ease of demonstration, the five states of damage considered for the bridge are light (at least one column subjected to ductility demand $\rho \geqslant 1$ ), minor (at least one column subjected to ductility demand $\rho \geqslant 2$ ), moderate (at least one column subjected to ductility demand $\rho \geqslant 3$ ), major (at least one column subjected to ductility demand $\rho \geqslant 4$ ) and collapse (at least one column subjected to ductility demand $\rho \geqslant 5$ ) under the longitudinal and transverse applications of ground motion.

Another set of five different damage states is also introduced following the Dutta and Mander [14] recommendations. Table 4 displays the description of these five damage states and the corresponding drift limits for a typical column. For each limit state in Table 4, the drift limit is transformed to peak ductility demand of the columns for the purpose of this study [7]. 


\subsection{Measure of ground motion intensity}

Expressing the fragility curves as functions of different measures of ground motion intensity has been advocated and promoted by many researchers and engineers. PGA, which is the absolute maximum value of the ground acceleration associated with a particular ground acceleration time history, has been mostly used to represent the ground motion intensity for fragility curves. However, SA, the maximum pseudo- (relative) response acceleration of a damped single-degree-of-freedom system to the ground acceleration, is the prominent one among these alternative measures. SA can be a good measure under the conditions that the structural response is primarily in the linear range, structural dynamic characteristics including damping properties are reasonably well known, geotechnically consistent earthquake ground motion time histories are either easily specificable or readily available from pertinent database, and the state of damage for which the fragility curve is to be developed depends mainly on the instantaneous maximum inertia force exerted by a ground motion time history. However, it is difficult to develop fragility curves on the basis of a large sample of structures subjected to severe earthquakes, since none of these conditions is satisfactorily met, particularly when the damage state of interest involves significantly non-linear structural deformation and/or the effect of repeated stress or strain cycle.

Some researchers also claim that ground velocity-related quantities including PGV, SV and SI are more appropriate for this purpose. PGV is the absolute maximum value of the ground velocity associated with a particular ground velocity, SV is the maximum pseudo- (relative) response velocity of a damped single-degree-of-freedom system to the ground acceleration, and SI is the average of SV over the natural period between 0.1 and $2.5 \mathrm{~s}$ following the original Housner's definition [15]. The structural damping coefficient is assumed in all calculations to be $5 \%$, although Housner [15] used $2 \%$ for SI calculations.

\subsection{Effect of spatial variation}

The fragility curves for the longitudinal direction of the bridge associated with those states of damage in Section 4.2 were plotted in Fig. 6a for the case without spatial variation and in Fig. 6b for the case with spatial variation as a functions of different measures of ground motion intensity including PGA, PGV, SA, SV and SI, in order to compare and highlight how ground motions with spatial variation affect structural behavior. The fragility curves for the transverse direction were also plotted in Figs. $7 \mathrm{a}$ and $\mathrm{b}$ in the same way. It is noted that the values of the ground motion intensity such as PGA, PGV, SA, SV and SI are different at different supports of the bridge for the case with spatial variation. For the purpose of practicality, these values are averaged and noted as mean PGA, mean PGV, mean SA, mean SV and mean SI in Figs. 6b and $7 \mathrm{~b}$. Fragility curves for the longitudinal direction, using another definition of damage states by Dutta and Mander [14] described in Section 4.2, were developed and plotted in Fig. 8. In Fig. 8a, the fragility curve for the collapse damage state is not available, since there is no such a case where a ductility demand exceeds 23.7 representing the state of collapse.

If the bridge damage is more susceptible to the ground motion with spatial variation than without it, the simulated fragility curves are at least consistent with the hypothesis that, for all levels of damage state, the median fragility values are larger without spatial variation than the corresponding values with spatial variation. The hypothesis is satisfied, however, only when the comparisons are made on the basis of the median ground motion intensity values measured in PGA for both longitudinal and transverse directions. SA, SV and SI produced similar results, whereas PGV mixed results. Further studies are needed to explore the reasons for these and other observations. One thing, however, is quite clear. If the number of bridges at a certain state of damage (e.g., at least light damage) is counted, it is larger when the entire sample is subjected to the ground motion with spatial variation than without it. In fact, the percentage of bridges subjected to the specific damage state for the 

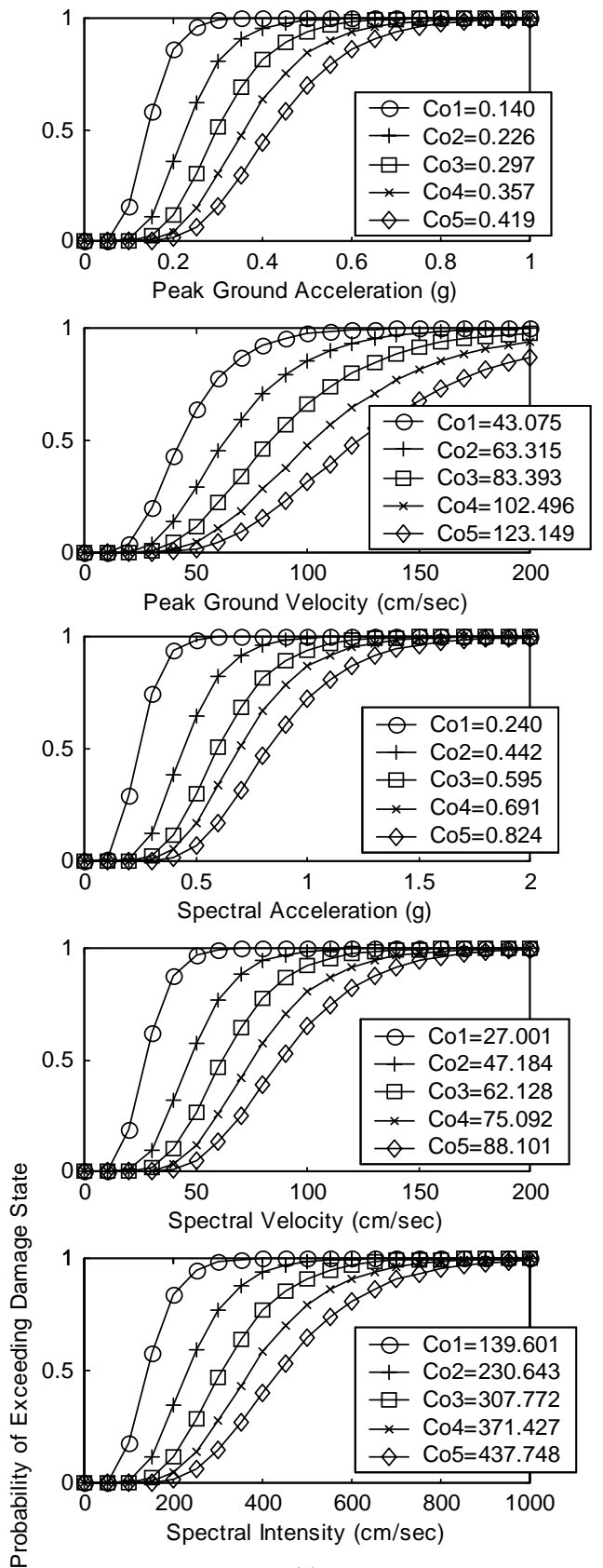

(a)
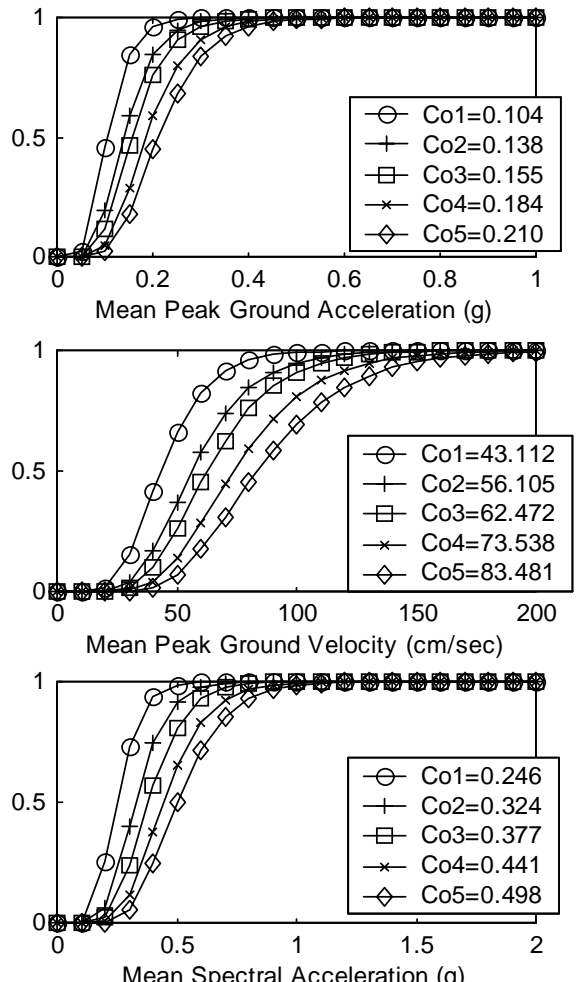

Mean Spectral Acceleration (g)

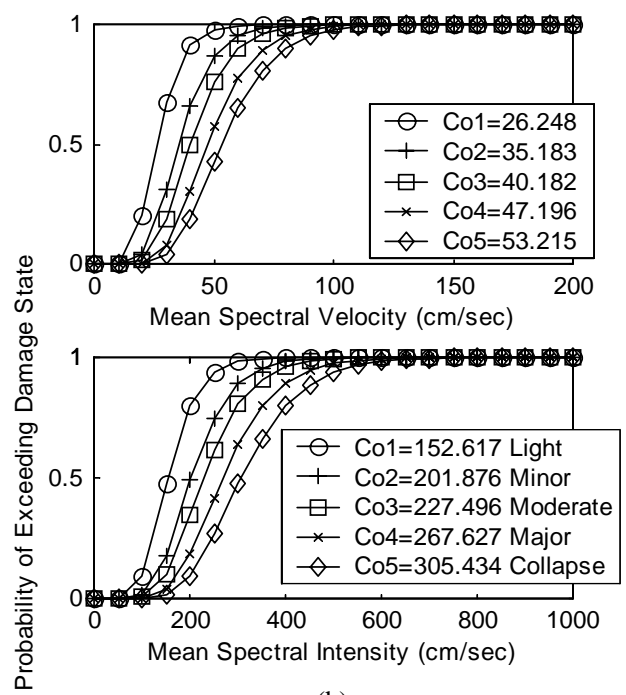

(b)

Fig. 6. Fragility curves for longitudinal direction of Santa Clara bridge. (a) Without spatial variation and (b) with spatial variation.

longitudinal and transverse directions of Santa Clara bridge under ground motion without and with spatial variation was listed in Table 5. Examining Table 5, it is found that the number of damaged bridges increases up to 2.3 times when the bridge is subjected to differential ground motion, compared to that under identical ground motion. 

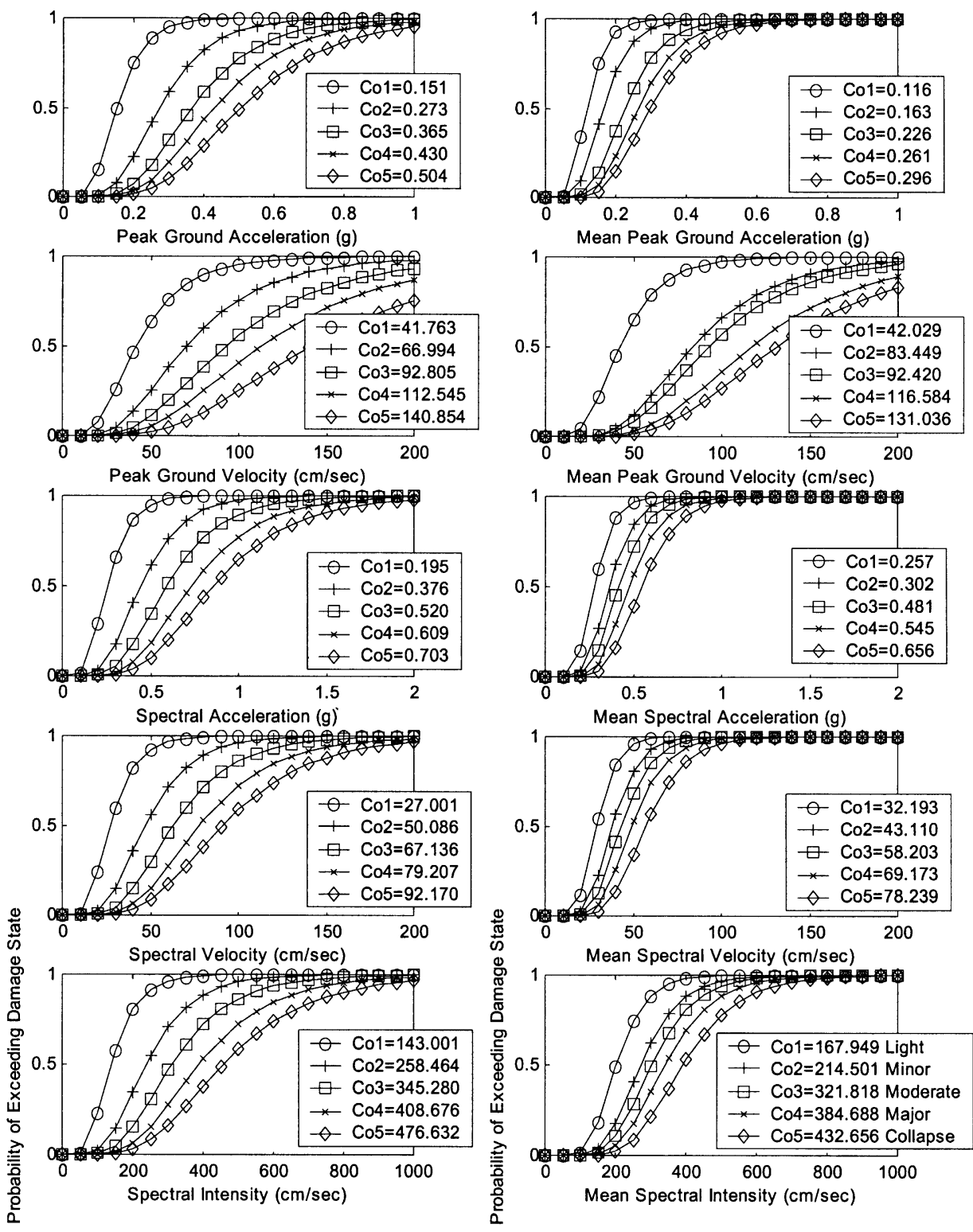

(a)

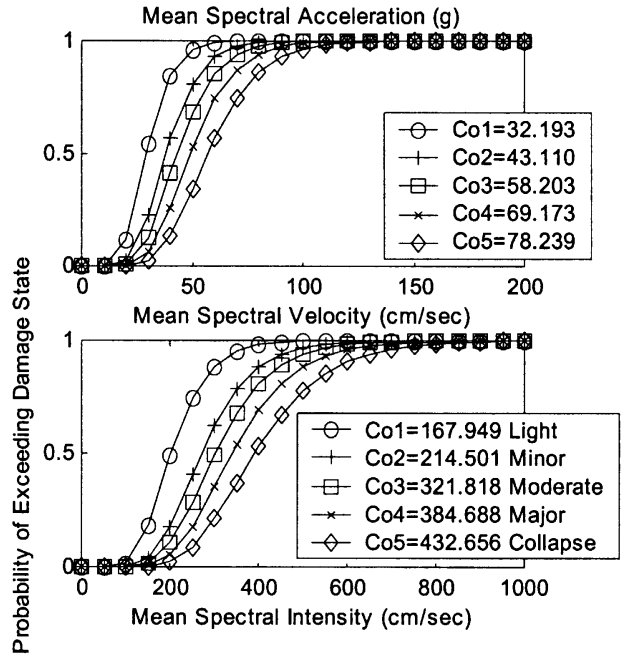

(b)

Fig. 7. Fragility curves for transverse direction of Santa Clara bridge. (a) Without spatial variation and (b) with spatial variation.

\section{Conclusions}

This paper presents fragility analysis of a bridge under ground motion with spatial variation. The analytical fragility curve is constructed for the Santa Clara bridge utilizing non-linear dynamic analysis to investigate the effect of spatial variation. Two-parameter log-normal distribution functions are used to represent the fragility curves utilizing the maximum likelihood procedure with each event of bridge damage treated as a realization from a multi-outcome Bernoulli-type experiment. In addition, some preliminary evaluations are made on the 

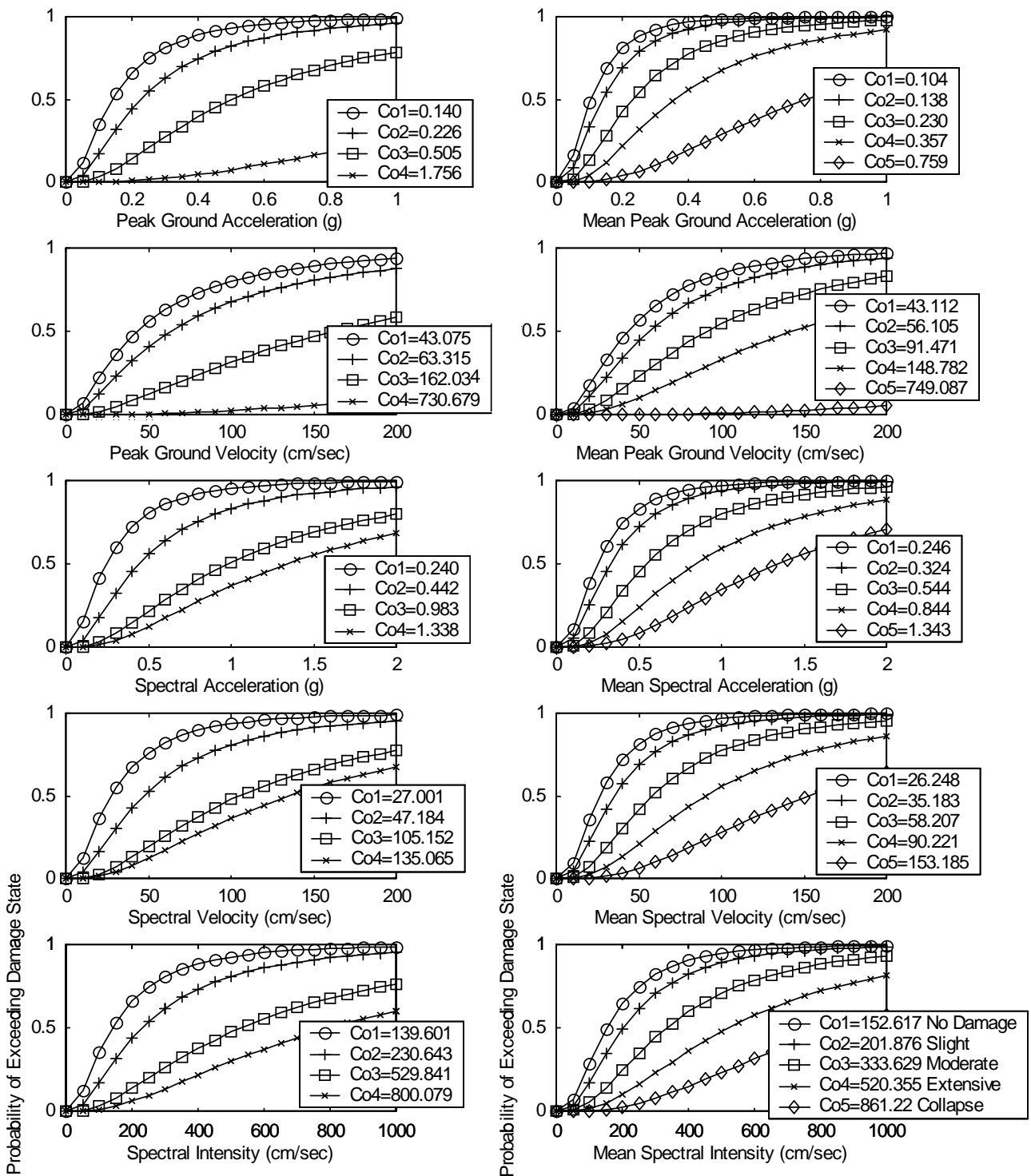

(a)

(b)

Fig. 8. Fragility curves for longitudinal direction of Santa Clara bridge using a set of five different damage states by Dutta and Mander [14]. (a) Without spatial variation and (b) with spatial variation.

significance of the fragility curves developed as a function of ground motion intensity measures other than PGA.

The computed fragility curves corresponding to these damage states appear to make intuitive sense relative to the bridge's design, construction, and performance in past seismic events. The following conclusions can be made on the results of this study.

(1) The simulated fragility curves obtained in this study are consistent with the hypothesis that the bridge is more vulnerable to the ground motion with spatial variation for all levels of damage state only when the 
Table 5

Percentage of damaged bridges ( sample size $=300$ )

\begin{tabular}{|c|c|c|c|c|}
\hline \multirow{2}{*}{$\begin{array}{l}\text { Damage } \\
\text { state }\end{array}$} & \multicolumn{2}{|l|}{ Longitudinal direction } & \multicolumn{2}{|l|}{ Transverse direction } \\
\hline & $\begin{array}{l}\text { Without spatial variation } \\
(\%)\end{array}$ & $\begin{array}{l}\text { With spatial } \\
\text { variation }(\%)\end{array}$ & $\begin{array}{l}\text { Without spatial variation } \\
(\%)\end{array}$ & $\begin{array}{l}\text { With spatial variation } \\
(\%)\end{array}$ \\
\hline Light & 84 & 88 & 80 & 92 \\
\hline Minor & 67 & 81 & 64 & 81 \\
\hline Moderate & 54 & 77 & 49 & 75 \\
\hline Major & 40 & 73 & 38 & 68 \\
\hline Collapse & 29 & 68 & 27 & 62 \\
\hline
\end{tabular}

comparisons are made on the basis of median ground motion intensity values measured in PGA. SA, SV and SI produced similar results, whereas PGV mixed results. Further studies are needed to explore these observations.

(2) For multi-span long bridges subjected to strong ground motion, the effect of spatial variation might increase the number of damaged bridges by as much as 2.3 times. Thus, a need is felt to take spatial variation into consideration for designing highway bridges.

\section{Acknowledgements}

The authors wish to acknowledge Dr. Saxena for providing the computer code for generating spatially varying ground motion time histories, the SAP2000 input file for the Santa Clara bridge and general advice on the development of the fragility curves. This study was supported by the National Science Foundation under grant CMS-9812585.

\section{References}

[1] N. Basoz, A.S. Kiremidjian, Evaluation of bridge damage data from the Loma Prieta and Northridge, California earthquake, Technical Report MCEER-98-0004, 1998.

[2] H. Hwang, J.B. Jernigan, Y.W. Lin, Expected seismic damage to Memphis highway systems, Proceedings of the Fifth US Conference on Lifeline Earthquake Engineering, Seattle, WA, USA, 1999.

[3] M. Shinozuka, M.Q. Feng, J.-H. Lee, T. Nagaruma, Statistical analysis of fragility curves, J. Eng. Mech. ASCE 126 (12) (2000) $1224-1231$.

[4] F. Yamazaki, T. Hamada, H. Motoyama, H. Yamauchi, Earthquake damage assessment of express bridges in Japan, Proceedings of the Fifth US Conference on Lifeline Earthquake Engineering, Seattle, WA, USA, 1999.

[5] I.G. Buckle, The Northridge, California earthquake of January 17, 1994: performance of highway bridges, Technical Report NCEER-94-0008, 1994.

[6] M. Shinozuka, G. Deodatis, V. Saxena, Effect of spatial variation of ground motion on bridge response, Technical Report MCEER-00-0013, 2000.

[7] V. Saxena, Spatial variation of earthquake ground motion and development of bridge fragility curves, Ph.D. Dissertation, Dept. of Civ. \& Envir. Engrg., Princeton University, Princeton, NJ, USA, 2000.

[8] G. Deodatis, V. Saxena, M. Shinozuka, Effect of spatial variability of ground motion on bridge fragility curves, Proceedings of the Eighth ASCE Specialty Conference on Probabilistic Mechanics and Structural Reliability, University of Norte Dame, 2000.

[9] V. Saxena, G. Deodatis, M. Shinozuka, M.Q. Feng, Development of fragility curves for multi-span reinforced concrete bridges, Proceedings of the International Conference on Monte Carlo Simulation, Principality of Monaco, Balkema Publishers, 2000. 
[10] G. Deodatis, Non-stationary stochastic vector processes: seismic ground motion applications, Probab. Eng. Mech. 11 (3) (1996a) 149-167.

[11] G. Deodatis, Simulation of ergodic multi-variate stochastic processes, J. Eng. Mech. ASCE 122 (8) (1996b) $778-787$.

[12] Computer and Structures, SAP2000N v.7.14 User manual, 1999.

[13] M. Shinozuka, M.Q. Feng, H.-K. Kim, T. Uzawa, T. Ueda, Statistical analysis of fragility curves, Unpublished Technical Report MCEER, 2001.

[14] A. Dutta, J.B. Mander, Rapid and detailed seismic fragility analysis of highway bridges, Unpublished Technical Report MCEER, 2001.

[15] G.W. Hausner, Intensity of ground motion during strong earthquakes, Proceedings of 1952 Symposium on Earthquake and Blast Effects on Structures, Earthquake Engineering Research Institute, California Institute of Technology, 1952. 